\title{
ANÁLISIS TRIMESTRAL DE LA COMPLEJIDAD LEGAL DE LA LEY DEL IMPUESTO AL VALOR AGREGADO EN EL PERIODO DE 1978 A 2016
}

QUARTELY ANALYSIS OF THE LEGAL COMPLEXITY OF THE VALUE ADDEA TAX LAW IN THE PERIODO FROM 1978 TO 2016

Javier Moreno* Jaime H. Beltrán** Leovardo Mata***

(Recibido: julio, 2016/Aceptado: noviembre, 2016)

\section{Resumen}

Se estudia la complejidad legal a través del modelo de Katz y Bommarito, de la ley del impuesto al valor agregado, desde su versión original publicada en 1978, hasta la versión vigente actualmente (2016). Los datos revelan que la ley se ha vuelto más compleja a través del tiempo, en términos de las variables estructura, entropía e interdependencia; utilizadas en el estudio para conformar el índice de complejidad.

Palabras clave: complejidad legal, impuesto al valor agregado. Clasificación JEL: E62.

\footnotetext{
Abstract

Through the Katz and Bommarito's model, we studied the legal complexity of the Value Added Tax Law, from its original version published on 1978,

*Candidato a doctor en Gestión Estratégica y Políticas de Desarrollo por la Universidad Anáhuac México Norte. Av. Universidad Anáhuac, núm. 46, col. Lomas Anáhuac, Huixquilucan, CP. 52786, Edo. de México. * Profesor-investigador del Instituto Tecnológico Superior de Pánuco. Prol. Av. Artículo Tercero Constitucional s/n, Col. Solidaridad, CP. 93990, Pánuco, Ver.

**** Posdoctorante en la Universidad Autónoma Metropolitana Iztapalapa. San Rafael Atlixco, núm. 108, Iztapalapa, col. Vicentina, 09340 Ciudad de México, México.
} 
until the current version (2016). The data reveals that the Law became more complex over time, in terms of structure, entropy and interdependence, the variables considered by this study for measuring legal complexity.

Keywords: legal complexity, value added tax.

JEL Classification: E62.

\section{Introducción}

La complejidad legal de una norma jurídica, en términos de lo establecido por el Internal Revenue Service, se entiende como su grado de ambigüedad o de falta de claridad; que en términos del presente trabajo, se refiere a la ambigüedad o falta de claridad de la ley del impuesto al valor agregado (LIVA), desde su versión original publicada en 1978 y su evolución en el tiempo, hasta el primer trimestre de 2016, como consecuencia de las modificaciones de las que ha sido objeto.

El modelo que se utiliza es el desarrollado por Katz y Bommarito (2013) para medir la complejidad legal de la LIVA, a partir del cual se construye un indicador que muestra la evolución de las modificaciones de la ley en el tiempo, lo que permite analizar la evolución que ha tenido el texto de la LIVA a lo largo del tiempo desde su publicación en 1978 hasta el año 2016. A partir de este indicador se podrá concluir si el marco legal es más complejo o menos complejo en términos de la estructura de la ley, del lenguaje utilizado y de la interdependencia de sus elementos.

Desde 1983, el impuesto al valor agregado ha sido la segunda mayor fuente de ingresos tributarios en México, representando en 2015 el 29.8\% del total de la recaudación anual. Desde su entrada en vigor (1980), la recaudación por concepto de IVA ha registrado una tasa media de crecimiento anual de $28.5 \%$, superior a la tasa de $26.5 \%$ de crecimiento anual promedio registrada por el total de ingresos tributarios durante el mismo periodo.

Entre 2008 y 2015 la recaudación del IVA registró una tasa media de crecimiento anual de $6.4 \%$, mientras que la recaudación de impuesto sobre la renta (ISR) y la recaudación total registraron tasas medias de crecimiento anuales de $11.9 \%$ y $13.2 \%$, respectivamente, durante el mismo periodo.

La recaudación de IVA en México durante 2012 representó el 3.8\% del producto interno bruto (PIB), cifra que contrasta con los niveles de $8.7 \%$, 
8.5\% y $8 \%$ registrados en Argentina, Brasil y Chile, respectivamente, durante el mismo año (OCDE, 2013).

Como puede apreciarse en la gráfica 1, la recaudación por concepto de IVA en México nunca ha rebasado el $4 \%$ como porcentaje del PIB; el punto más alto se registró en 2010, año en que la recaudación de IVA representó el $3.85 \%$ como proporción al PIB.

\section{Gráfica 1}

Recaudación de IVA como porcentaje del PIB

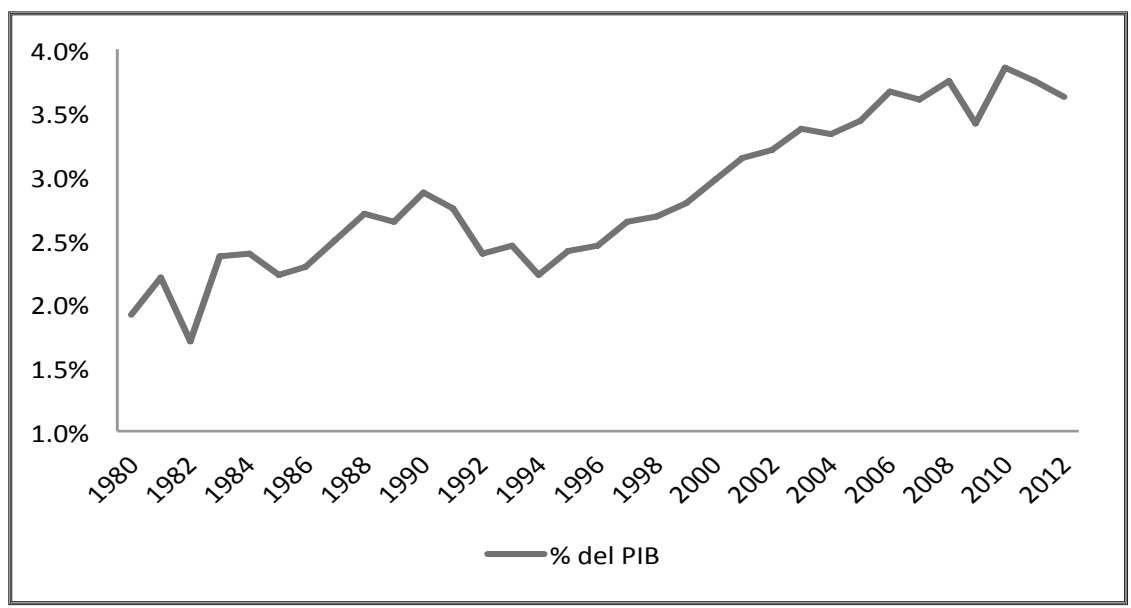

Fuente: elaboración propia con datos de Centro de Estudios de las Finanzas Públicas.

Debido a la relevancia económica del IVA en los ingresos del Estado es de gran valor para la ciencia de las finanzas públicas identificar variables adicionales al crecimiento económico que contribuyan a mejorar la eficiencia de la recaudación de este impuesto.

De lo anterior se deriva la hipótesis que estipula que una mayor complejidad en la ley que regula el impuesto al valor agregado tiene efectos negativos en los niveles de recaudación por concepto de dicho impuesto, argumento que concuerda con lo estipulado por Kades (1997).

El objetivo general de esta investigación es crear un índice capaz de medir la evolución de la complejidad de la LIVA desde su publicación en 1978 hasta 2016, que permita profundizar en el estudio de la relación entre los niveles de complejidad y la recaudación por concepto de IVA. 
Se pretende identificar una variable -adicional al crecimiento económicoque contribuya a rebasar, en primera instancia, el umbral del $4 \%$ de recaudación de IVA como proporción del PIB.

Los resultados permitirán sentar las bases para demostrar que un alto nivel de complejidad en las leyes genera efectos negativos en los niveles recaudatorios y de cumplimiento de obligaciones fiscales, situación que implica una pérdida económica para el Estado.

\section{Antecedentes}

El estudio de la complejidad legal se remonta a los años setenta, cuando en 1972, un grupo de expertos fiscalistas de la American Bar Association $(\mathrm{ABA})^{1}$ analizó la complejidad legal del Código Fiscal de Estados Unidos, concluyendo que la complejidad de las leyes fiscales se debía a la participación de múltiples actores implicados en este tema. Este trabajo sirvió de base para futuras investigaciones respecto de la complejidad legal en otros ámbitos (Roberts, Friedman, Ginsburg y Young, 1972).

Long y Swingen (1987) estudiaron la complejidad del Código Fiscal de los Estados Unidos y concluyeron que su investigación se basó en una muestra pequeña de opiniones y experiencias de profesionales en temas fiscales, y que resultaba necesaria mayor profundidad en la investigación para conocer cómo el nivel de ingresos de cada contribuyente influye en la complejidad para comprender la ley fiscal.

La obra "Legal complexity: some causes, consequences and cures" -de Peter Schuck (1992)- representó uno de los primeros trabajos en abordar el tema de la complejidad legal, definiendo a un sistema como complejo "cuando sus reglas, procesos e instituciones presentan cuatro características: densidad, tecnicismos, diferenciación e incertidumbre". El análisis de Schuck permitió identificar a la ley fiscal como un instrumento legal con un alto grado de complejidad.

Eric Kades (1997) utilizó la teoría matemática desarrollada por expertos informáticos para estudiar la complejidad de una Ley e identificó los límites

\footnotetext{
${ }^{1}$ La American Bar Association es una sociedad de abogaos de membresía voluntaria de Estados Unidos que no está sujeta a ninguna jurisdicción estatal específica.
} 
de la capacidad humana al aplicar normas jurídicas a situaciones cotidianas. Este autor afirmó que "el mejor ejemplo de complejidad legal podría encontrarse en la ley fiscal".

Epstein (1997) definió la complejidad legal en términos del costo de cumplimiento; es decir, si la norma es sencilla el costo de cumplir deberá ser menor. El argumento primordial del autor es que una ley se considera compleja en la medida que genere obstáculos regulatorios que dificulten el logro de algún objetivo privado. Epstein rechazó categóricamente el argumento de que una sociedad compleja requiere incrementar el alcance de las leyes y estableció que las leyes complejas son para las sociedades que tienen una visión inalcanzable de la perfección.

Bourcier y Mazzega (2007) explicaron la complejidad legal como un sistema dinámico complejo compuesto por estructuras interconectadas; utilizaron dinámicas no lineales existentes en cualquier sistema legal, y propusieron dos modelos de medición de complejidad: un modelo basado en la estructura de la ley, y otro basado en el contenido y densidad del cuerpo legal. Los autores concluyeron que los factores que más influyen en la complejidad legal son: el número de componentes y la interacción entre ellos, el conocimiento parcial de los nexos dinámicos entre componentes, una limitada previsibilidad de la evolución del sistema legal, y la adición de nuevos componentes a una ley.

Daniel Katz (2010) desarrolló un modelo basado en principios estadísticos y matemáticos para analizar el Código de Estados Unidos, con la finalidad de dar una mayor objetividad al estudio de la complejidad legal.

A partir de sus trabajos de 2010 Daniel Katz junto con Michael Bommarito desarrollaron en 2013 un indicador matemático que busca medir el concepto de complejidad legal a través de la ponderación de tres aspectos: la estructura, el lenguaje y la interdependencia del código de leyes de los Estados Unidos.

El indicador de Katz y Bommarito (2013) mide la dificultad que enfrenta un individuo estándar al momento de buscar una buena comprensión del marco legal, bajo la premisa de que a mayor complejidad, mayor costo de cumplimiento. Concluyeron que una innecesaria complejidad legal puede provocar una mala asignación del capital humano para la comprensión y cumplimiento de las normas.

Ruhl y Katz (2014) realizaron una distinción con respecto a la complejidad en el sentido de la confusión o complicación que experimenta un individuo 
al entender una ley, y la complejidad con respecto a la estructura y el comportamiento del cuerpo legal; explicaron que la complejidad legal aumenta cuando las dependencias entre los elementos de una ley se incrementan, debido a que una modificación en un elemento lo afecta a sí mismo y a los elementos a los que hace referencia.

Waltl y Matthes (2014) realizaron un análisis de las propiedades estructurales, léxicas y sintácticas en más de tres mil leyes alemanas utilizando métodos cuantitativos de análisis estructural de las conexiones entre elementos de la ley y la lingüística. Waltl y Matthes calcularon diversos indicadores estructurales de léxico y de correlaciones $y$, concluyeron que es posible demostrar que la duración media de los párrafos de una ley se correlaciona con la legibilidad y, por lo tanto, con su nivel de complejidad.

Krever y Mellor (2015) estudiaron la complejidad legal de la Ley Fiscal de Estados Unidos definiéndola como el costo de cumplimiento de la norma y propusieron modificar las leyes para facilitar su comprensión y cumplimiento.

A partir de los antecedentes enunciados se concluye que existe consenso respecto a la existencia de una relación entre la complejidad legal y el cumplimiento de las obligaciones fiscales. Cuando un contribuyente enfrenta mayor complejidad en el entendimiento y comprensión de una ley, condiciona su decisión de cumplir o no cumplir con sus obligaciones fiscales, con su consecuente impacto en la recaudación de un impuesto. El consenso identificado en la literatura es la principal motivación de los autores para evaluar la complejidad legal de la LIVA, con el fin de identificar si la complejidad legal tiene impacto - positivo o negativo- en la recaudación del IVA.

Debido a que la investigación que se realiza sobre la posible correlación entre complejidad legal y la recaudación del IVA, se requiere un indicador cuantitativo que mida la complejidad de la LIVA a lo largo del tiempo, para lo cual se determinó adecuar el enfoque propuesto por Katz y Bommarito (2013).

\section{Construcción del indicador de complejidad legal}

Para medir la complejidad de la ley del IVA a lo largo del tiempo se requiere un indicador cuantitativo que mida esta variable, por lo que se determinó adecuar el enfoque propuesto por Katz y Bommarito (2013) para considerar los componentes: estructura, lenguaje e interdependencia interna y con otras leyes. 
Los autores del modelo que se utiliza (Katz y Bommarito), realizaron una comparación entre diversos instrumentos legales, por lo que fue modificado el modelo debido a que esta investigación estudia únicamente la evolución en el tiempo de la complejidad de la ley del impuesto al valor agregado desde 1978² hasta 2016; no es materia de esta investigación analizar la interdependencia de otras leyes con la LIVA.

\subsection{Estructura de la ley}

La variable estructural es un componente del indicador de complejidad legal y representa la forma en que está organizada la ley a partir de la cual se identifica la profundidad de los elementos que la integran; es decir, artículos, párrafos, fracciones, incisos y numerales. La construcción de este subíndice se realiza dando un valor mayor conforme a la profundidad de cada elemento; es decir, se asigna un valor de 2 a cada párrafo, y un valor de 5 a cada numeral.

\section{Tabla 1}

Valor por tipo de elemento

\begin{tabular}{lc}
\hline Tipo de referencia & Valor por referencia \\
\hline \hline artículo & 1 \\
párrafo & 2 \\
fracción & 3 \\
inciso & 4 \\
numeral & 5 \\
\hline
\end{tabular}

Fuente: elaboración propia.

Este método permite identificar los momentos/periodos en los que la ley se vuelve más profunda, debido a la adición de elementos más específicos (incisos o numerales, por ejemplo), o menos profunda, cuando se suprimen o derogan estos elementos.

\footnotetext{
${ }^{2}$ La ley del impuesto al valor agregado fue publicada en el Diario Oficial de la Federación en 1978, su entrada vigor fue en 1980 .
} 


\subsection{Lenguaje de la Ley}

La variable lenguaje se analiza desde la óptica de la entropía (S), en concordancia con el modelo desarrollado por Katz y Bommarito, lo que busca definir las veces que cada palabra se repite a lo largo del texto de la ley (frecuencia $p_{w}$ ).

Para el cálculo de la entropía, se utilizaron técnicas de minería de texto, a partir de las cuales se obtuvo la frecuencia de las palabras que aparecen en la LIVA de todas las versiones publicadas de este ordenamiento legal desde 1978 hasta 2016. Los datos obtenidos permitieron calcular la entropía para cada una de las palabras, de acuerdo a la siguiente fórmula:

$$
S=-\sum_{w \in W} p_{w} \log _{2}\left(p_{w}\right)
$$

El valor de entropía para cada palabra toma valores entre 0 y 1 ; cuando el valor es cero se deberá entender como un texto sin entropía; es decir, la distribución de ese texto es completamente uniforme. Cuando el valor de este indicador es cercano a uno, se refiere a un texto con una distribución más compleja o una mayor cantidad de palabras.

Una vez calculada la entropía de todas las palabras que conforman la ley se realiza una sumatoria de los valores obtenidos, resultando el nivel general de entropía de la LIVA en determinado año.

\subsection{Interdependencia de la ley}

En la presente investigación, a diferencia del modelo desarrollado por Katz y Bommarito, se realiza una distinción entre referencias internas y externas; se consideran como referencias internas a aquellas en las que un elemento se refiere a cualquier otro elemento dentro de la LIVA, mientras que las referencias externas son aquellas que citan elementos externos a la ley en cuestión.

Las referencias se clasifican en cuatro tipos: las citas al párrafo anterior, las citas a algún apartado dentro de la misma LIVA, las citas a artículos específicos de otras leyes y las citas a otras leyes de forma genérica, sin especificar artículo o texto al que se debe dirigir el lector. En todos los casos se parte del supuesto que conlleva menor costo de adquisición de conocimiento cuando se trata de una cita a un párrafo anterior que cuando se cita una ley distinta sin especificar explícitamente la referencia que se está realizando. 
Con base en lo anterior, a cada referencia encontrada en el texto se le asigna un valor, dependiendo de su categoría, con el fin de representar el grado de complejidad que significa buscar y entender el referente:

Tabla 2

Valor por tipo de referencia

\begin{tabular}{lc}
\hline \multicolumn{1}{c}{ Tipo de referencia } & Valor por referencia \\
\hline \hline párrafo anterior & 1 \\
elemento dentro de la ley del IVA & 2 \\
elemento específico de otra ley & 3 \\
referencia general a otra ley & 4 \\
\hline \hline
\end{tabular}

Fuente: elaboración propia.

En concordancia con la metodología utilizada hasta este punto, el análisis de referencias es realizado en todos los elementos que componen la LIVA; a cada referencia encontrada a lo largo del texto se le asignó el valor correspondiente de acuerdo a su clasificación, posteriormente se realizó una sumatoria de los valores obtenidos, dando como resultado el nivel general de interdependencia de la ley.

\subsection{Indicador de complejidad legal conjunto}

Una vez obtenidos los valores de los tres componentes -estructura, lenguaje e interdependencia-, se construyó el indicador de complejidad legal, con base en dos metodologías: con una sumatoria de los valores absolutos de los tres subíndices, y con un método de sumatoria de los logaritmos naturales de los valores absolutos de los tres subíndices.

\subsubsection{Indicador de complejidad con valores absolutos}

El indicador de complejidad legal estimado con valores absolutos es obtenido a partir de la sumatoria de las cantidades obtenidas para cada uno de los componentes que conforman la complejidad:

$$
\text { complejidad }=\mathrm{E}+S+\operatorname{Ref}
$$


Resulta importante destacar que este método considera los valores tal y como son calculados; es decir, suma los productos de estructura (que van desde 278 a 681), los de entropía (que van de 8.57 a 8.92) y los de interdependencia (que van desde 61 hasta 529) veáse anexo.

\subsubsection{Indicador de complejidad con logaritmos naturales}

El indicador de complejidad legal estimado con logaritmos naturales es obtenido de la sumatoria de los logaritmos naturales de los valores obtenidos para cada subíndice:

$$
\text { complejidad }=\ln E+\ln S+\ln R e f
$$

Este método permite dar una ponderación más uniforme a los valores de los tres elementos que componen el índice: estructura (de 5.62 a 6.52), entropía (de 2.14 a 2.18) e interdependencia (de 4.11 a 6.27).

\section{Evaluación de la complejidad legal de la LIVA}

Para obtener el indicador de complejidad legal de la LIVA se obtienen los indicadores parciales de estructura, lenguaje e interdependencia de cada una de las versiones de la ley bajo estudio; a partir de ello, se procede a determinar el indicador de complejidad conjunto.

\subsection{Evaluación de la estructura de la LIVA}

La evaluación anual de la estructura de la LIVA desde su versión original (1978) hasta la versión vigente (2016) se realizó a partir de enlistar cada uno de sus elementos (todos sus párrafos, fracciones, incisos y numerales), para posteriormente ser ponderados de acuerdo a su nivel de especificidad.

En la gráfica 2 se muestra que la LIVA, en su versión original (1978) tenía un nivel de estructura de 278 puntos (con 173 elementos). La LIVA de 2016 muestra un nivel más complejo en su estructura, con un nivel de 621 puntos (con 340 elementos), más del doble que la versión original de 1978, tanto en el subíndice ponderado de estructura, como en número de elementos. 


\section{Gráfica 2}

Nivel de complejidad en la estructura de la ley del IVA (trimestral, 1978-2016)

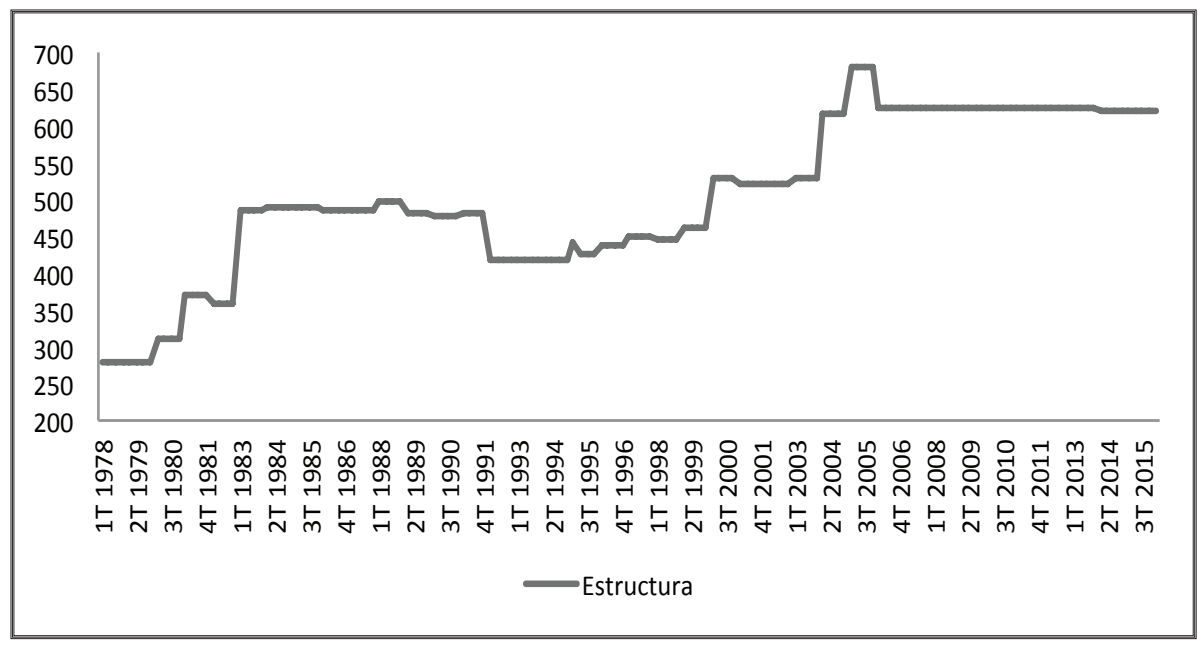

Fuente: elaboración propia.

La versión más compleja de la LIVA estuvo vigente durante el tercero y cuarto trimestre de 2005, que registró un nivel de complejidad en su estructura de 681 puntos (357 elementos).

La evaluación realizada permite identificar que, en términos generales, la ley del IVA ha sido objeto de una mayor cantidad de adiciones que derogaciones desde su publicación. Al respecto destaca la versión vigente a partir del primer trimestre de 1983, en la que se presentó el mayor cambio en el valor de complejidad en la estructura (126 puntos más con respecto a la versión que antecede).

A continuación, se presentan los dendrogramas de la estructura de la LIVA en dos momentos específicos: la versión original de 1978 (gráfica 3) y la ley vigente en 2016 (gráfica 4). 
Gráfica 3

Estructura de la ley del IVA (1978)

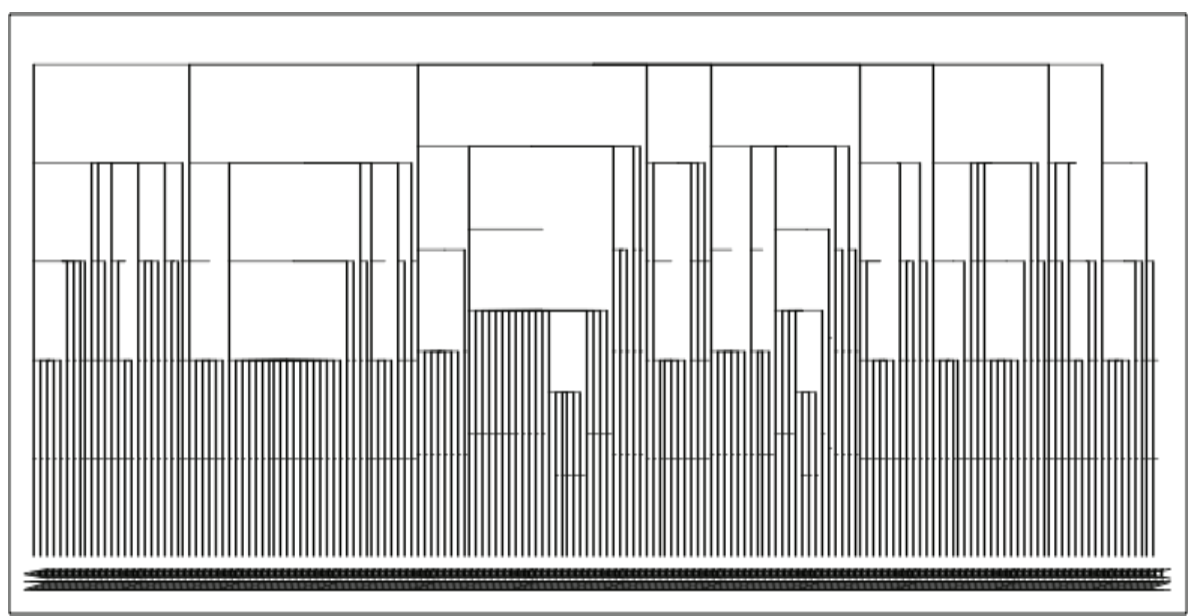

Fuente: elaboración propia.

Gráfica 4

Estructura de la ley del IVA (2016)

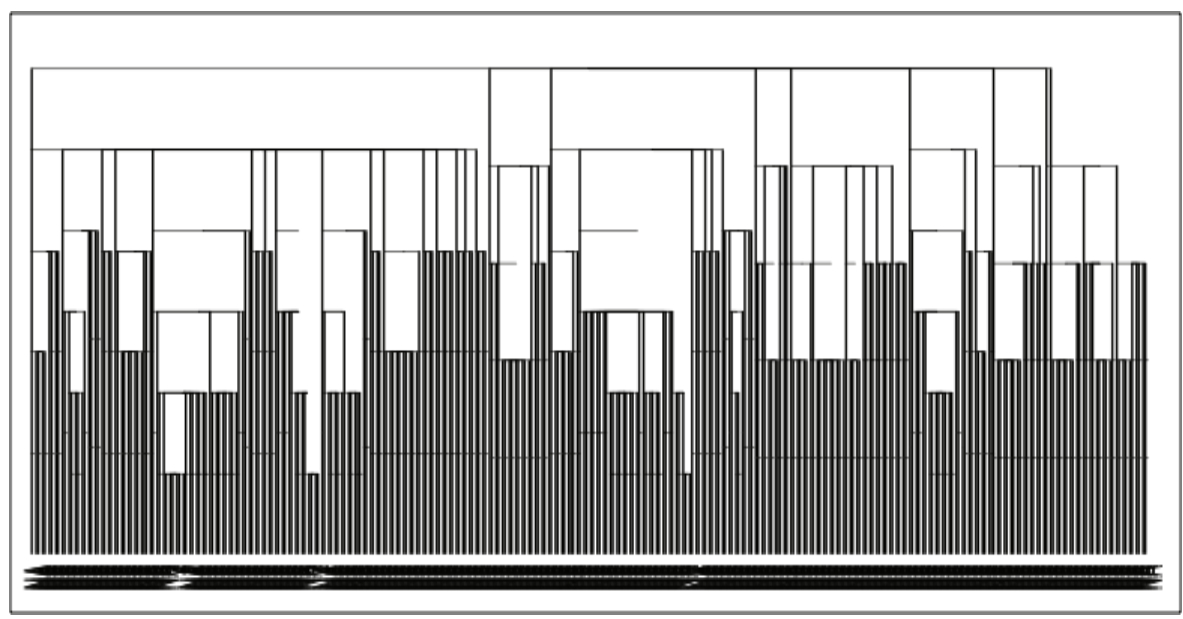

Fuente: elaboración propia. 
Los dendrogramas anteriores expresan una mayor densidad de los elementos y profundidad en la ley del IVA vigente en 2016, con respecto a la ley publicada en 1978. Con la finalidad de hacer más explícito el nivel de complejidad en la estructura de la ley, los diagramas anteriores también pueden ser representados mediante la gráfica 5 .

\section{Gráfica 5}

Estructura de la ley del IVA

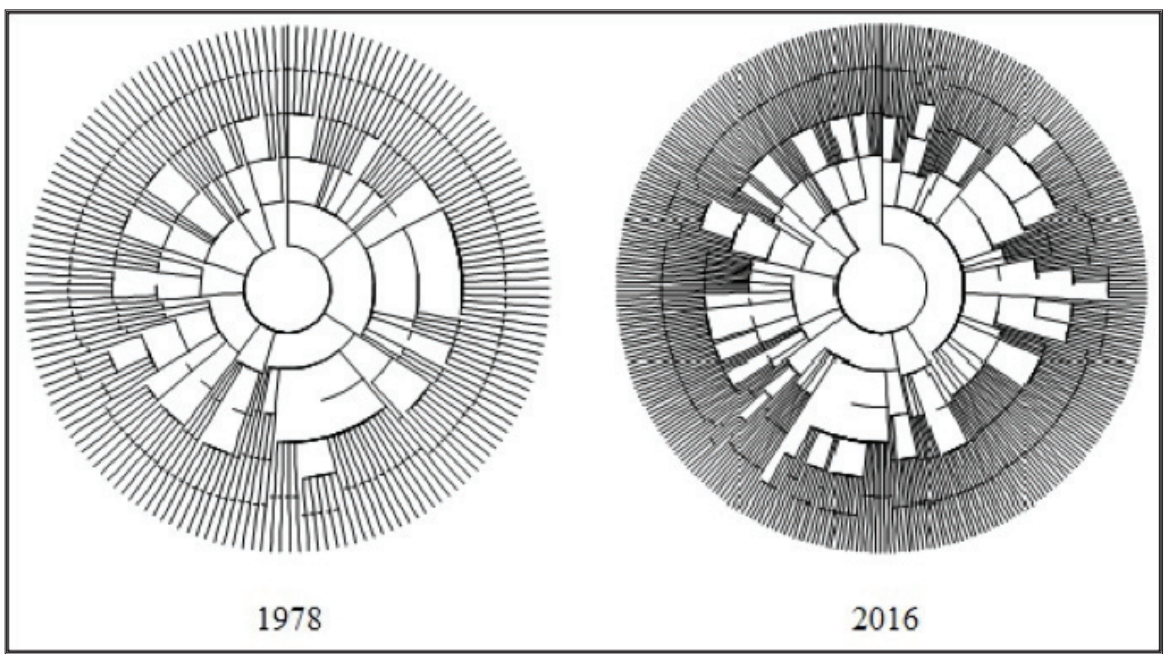

Fuente: elaboración propia.

\subsection{Evaluación del lenguaje de la LIVA}

La entropía de las palabras se calculó mediante la utilización de herramientas de minería de texto, que permitió identificar el total de palabras en cada versión de la LIVA bajo estudio, así como su frecuencia a lo largo del texto.

El nivel de entropía de la LIVA se obtuvo sumando los valores obtenidos de cada palabra, dada su distribución en la versión de ley en la que se encuentra. La entropía general de la Ley se obtuvo sumando los valores de entropía de todas las palabras que conforman la LIVA.

En la gráfica 6 se aprecia que el nivel de entropía de la Ley del IVA vigente es cuatro décimas más grande con respecto a la ley publicada en 1978, una cantidad que podría parecer menor en términos absolutos, pero que en 
realidad expresa una adición de más de 800 palabras distintas entre ambas versiones.

\section{Gráfica 6}

Nivel de entropía de la ley del IVA

(trimestral, 1978-2016)

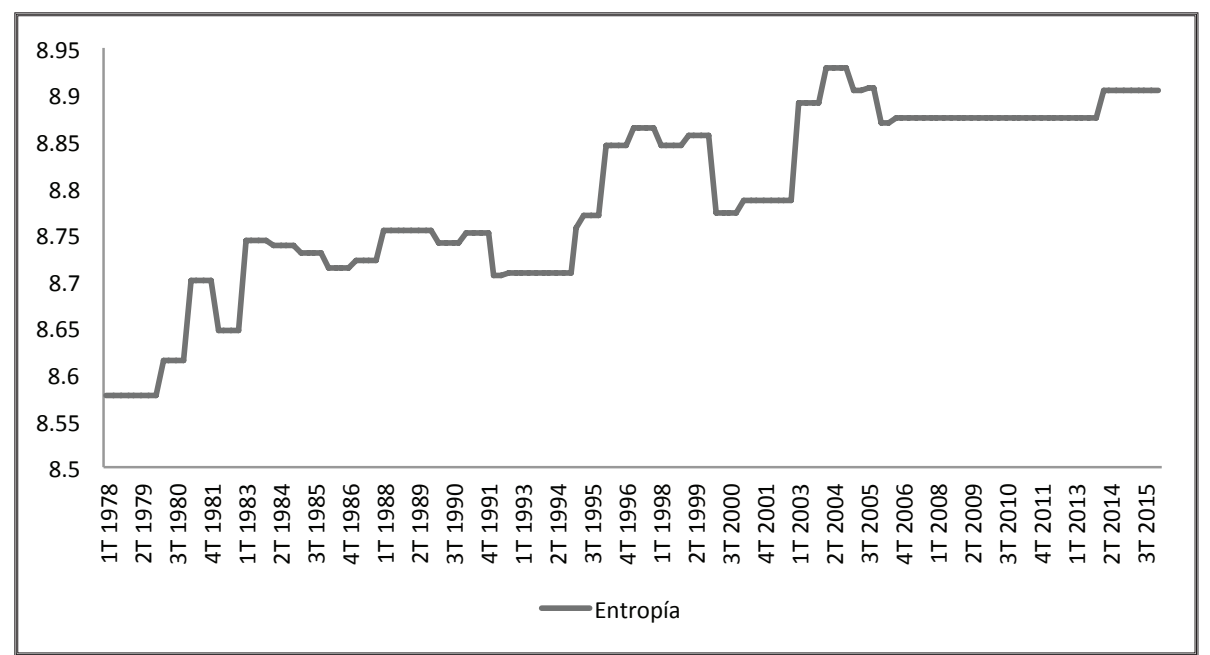

Fuente: elaboración propia.

La versión de 1978 tenía una extensión de 3878 palabras (utilizando 1078 palabras distintas), mientras que la ley vigente (2016) cuenta con una extensión de 11355 palabras (utilizando 1926 palabras distintas), representando una ampliación de la ley de casi tres veces su extensión original (equivalente a adicionar a la ley en promedio 196 palabras cada año).

\subsection{Evaluación de la interdependencia de la LIVA}

El cálculo de interdependencia para cada versión de la ley bajo estudio, se realizó sumando los valores que resultan de la multiplicación del ponderador definido en el modelo por cada una de las referencias identificadas a lo largo del texto, para cada una de las versiones de la ley del IVA.

La gráfica 7 permite identificar que el nivel de interdependencia en la ley vigente (2016) es siete veces mayor que el nivel de interdependencia registrado originalmente (1978). 


\section{Gráfica 7}

Nivel de interdependencia de la ley del IVA

(trimestral, 1978-2016)

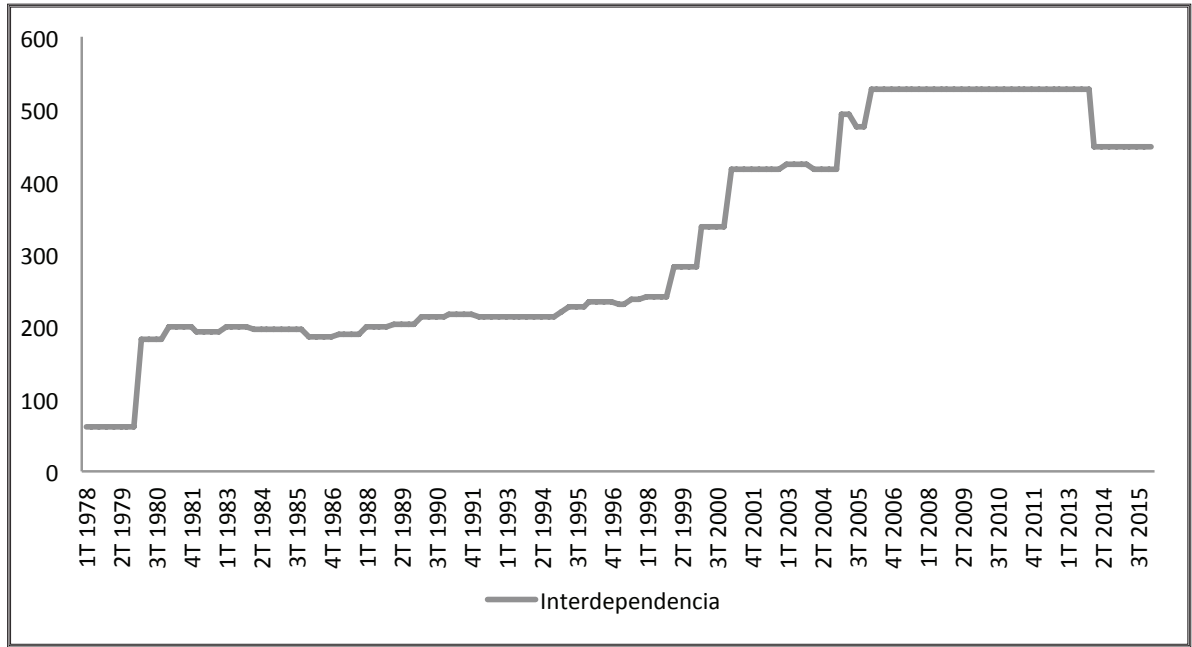

Fuente: elaboración propia.

El aumento más significativo en el nivel de interdependencia se presenta en la versión vigente a partir del primer trimestre de 1980 (122 puntos mayor con respecto a la versión que antecede); al respecto destaca que esta adición de referencias a la LIVA se presentó justo en el momento de su entrada en vigor, apenas dos años después de haber sido publicada.

\section{Resultados del cálculo del indicador de complejidad legal en la ley del IVA}

\subsection{Valores absolutos}

Una vez obtenidos los valores correspondientes a cada componente, es posible construir el índice de complejidad legal con base en la suma de dichos valores:

$$
\text { complejidad }=\mathrm{E}+S+\operatorname{Ref}
$$


El cálculo anterior, realizado a todas las versiones de la ley del IVA, muestra los siguientes resultados:

El nivel de complejidad de la ley del IVA pasó de 347.5 puntos en su versión original (1978) a 1077.9 en la versión vigente (2016), como consecuencia de los incrementos en los tres componentes analizados de estructura, entropía e interdependencia.

El nivel de estructura de la LIVA pasó de 278 en 1978 a 621 en el primer trimestre de 2016, siendo éste el componente con mayor peso en la ponderación del índice de complejidad legal con valores absolutos.

Este resultado fue consecuencia de las adiciones y modificaciones a la ley, lo que incrementa la dificultad de comprensión del instrumento legal, que en palabras de Bourcier y Mazzega (2007), el cambio en los componentes de una ley incrementan su complejidad.

En la primera versión de la LIVA, el nivel de entropía fue de 8.57, mientras que en el primer trimestre de 2016 registró un nivel de 8.9; esto se debió a la inclusión de aproximadamente 200 palabras por año a lo largo de 37 años de vigencia.

El nivel de interdependencia de la LIVA pasó de 61 en 1978 a 448 en el primer trimestre de 2016, siendo éste el componente con mayor crecimiento relativo desde que esta ley entró en vigor (en 2016 representa más del 600\% que el nivel registrado en la versión de 1978), lo que reafirma el concepto expresado por Bourcier y Mazzega (2007), referente a que la interdependencia entre elementos es lo que mayor ambigüedad genera en la comprensión de una ley.

En la gráfica 8 se observa el nivel general de complejidad de la LIVA, en donde destaca que el nivel más alto de complejidad legal se registró durante el primer y segundo trimestre de 2005, con 1179.9 puntos.

Resulta importante indicar que en este análisis con valores absolutos, los cambios en la entropía de la ley (que implica la adición de 7477 palabras referida anteriormente) tienen poca influencia sobre el nivel general de complejidad legal. 


\section{Gráfica 8}

Complejidad de la ley del IVA en valores absolutos

(trimestral, 1978-2016)

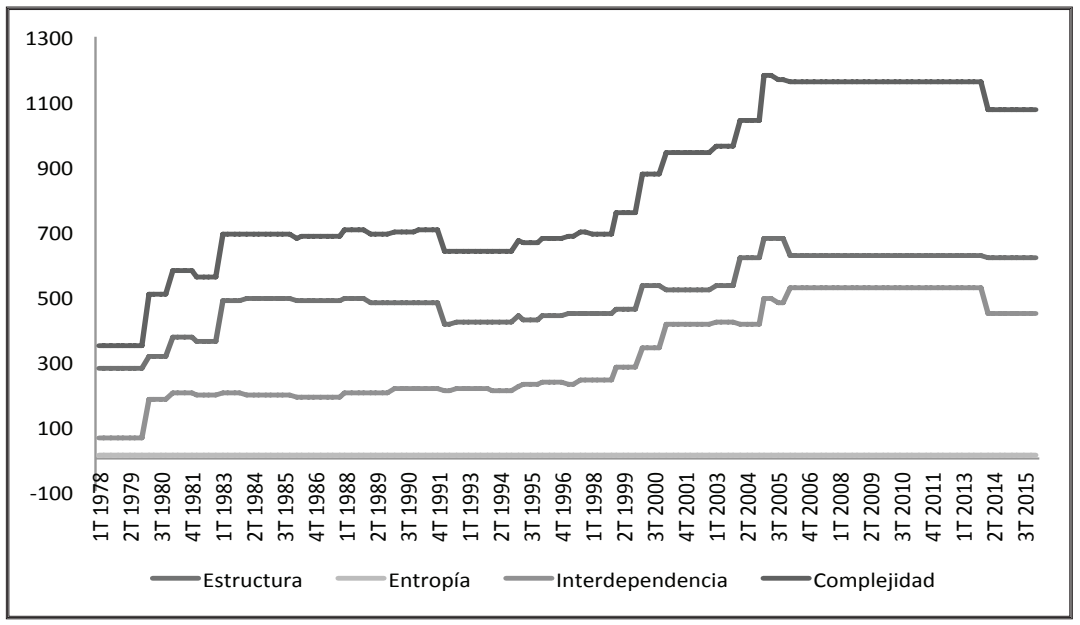

Fuente: elaboración propia.

\subsection{Valores con logaritmos naturales}

Este índice de complejidad legal es construido a partir de la suma de los logaritmos naturales de los valores correspondientes a cada subíndice, con el objeto de suavizar el comportamiento de los valores absolutos, dada la fórmula:

$$
\text { complejidad }=\ln E+\ln S+\ln R e f
$$

El cálculo anterior, realizado a todas las versiones de la ley del IVA, muestra los siguientes resultados:

En valores logarítmicos, la complejidad legal de la ley del IVA pasó de ser 11.88 en su versión original de 1978, a 14.72 en la versión vigente (2016), encontrando su nivel más alto durante el primer y segundo trimestre de 2005.

En el componente de estructura la LIVA pasó de 5.62 en 1978 a 6.43 en el primer trimestre de 2016; es decir, registró un incremento de $14.41 \%$ en valores logarítmicos, como resultado de una mayor cantidad de adiciones que derogaciones a lo largo del tiempo. 
En la primera versión de la LIVA el nivel de entropía fue de 2.14, mientras que en el primer trimestre de 2016 registró un nivel de 2.18. En valores logarítmicos la entropía tiene una participación porcentual del 14.66\% (en 2010) y $18.08 \%$ (en 1978) del nivel general de complejidad legal. La entropía es el componente que menos explica la evolución de la complejidad legal de la ley.

El nivel de interdependencia de la LIVA pasó de 4.1 en 1978, a 6.1\% en el primer trimestre de 2016; es decir, registró un incremento de $48.8 \%$ en valores logarítmicos, como resultado de una mayor cantidad de referencias a lo largo del texto de la ley.

En la gráfica 9 es posible observar el nivel general de complejidad de la LIVA (amarillo), que se obtiene de la suma de los logaritmos naturales de los valores que la conforman: estructura, entropía e interdependencia.

Gráfica 9

Complejidad de la ley del IVA con logaritmos naturales

(trimestral, 1978-2016)

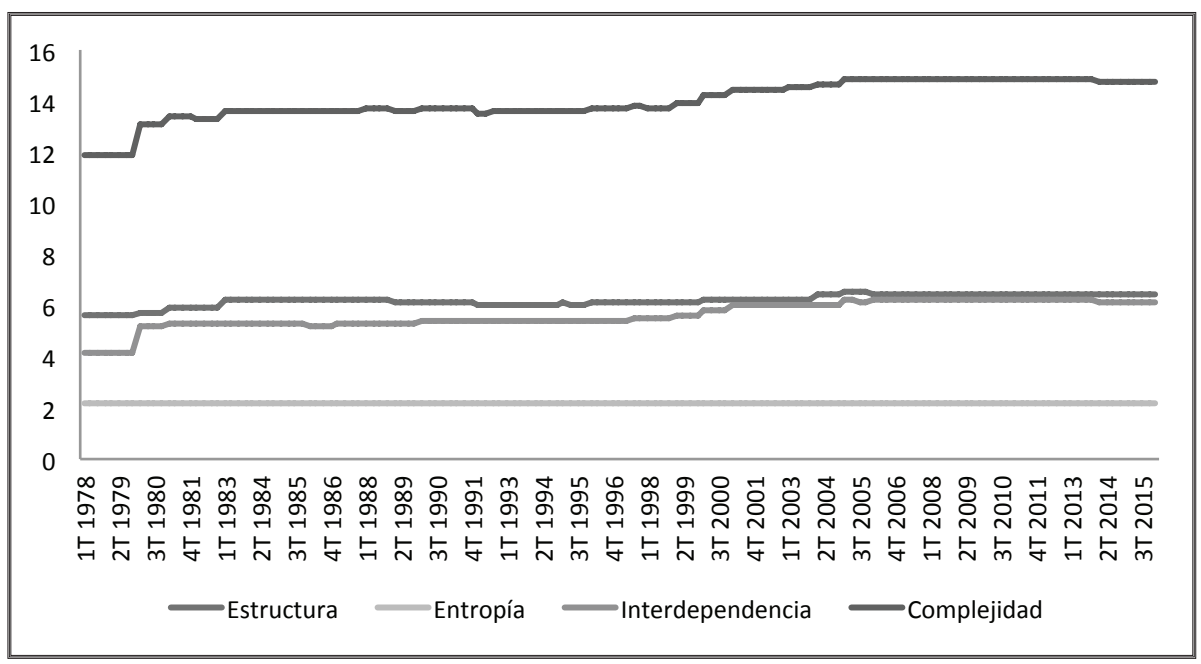

Fuente: elaboración propia.

En resumen, la complejidad legal en una escala logarítmica ha crecido en $24 \%$ a lo largo de 37 años de vigencia; resultado que es coincidente con el indicador de complejidad legal en valores absolutos. Este crecimiento ha sido resultado principalmente de las modificaciones a la estructura e interdependencia en el texto de la ley. 


\section{6. Índice de correlación entre variables}

La correlación entre las partes que conforman el indicador de complejidad legal, medida a través del coeficiente de correlación de Pearson es significativa, lo que significa que las partes están relacionadas de forma directa; es decir, que cuando una sufre cambios, las otras dos presentan movimientos positivos en su comportamiento.

El coeficiente de correlación de Pearson estipula que un valor entre 0 y 1 se refiere a que dos conjuntos de datos están correlacionados positivamente; es decir, un incremento en una variable provoca un incremento en la variable relacionada (McFedries, 2010).

\section{Tabla 3}

Índice de correlación entre variables

(coeficiente de Pearson)

\begin{tabular}{lccc}
\hline & Estructura & Entropía & Interdependencia \\
\hline \hline estructura & 1 & 0.876 & 0.902 \\
entropía & 0.876 & 1 & 0.837 \\
interdependencia & 0.902 & 0.837 & 1 \\
\hline \hline
\end{tabular}

Fuente: elaboración propia.

El alto grado de correlación entre las variables de estudio se explica, en gran medida, debido a que una modificación en cualquiera de ellas, implica también un cambio en las variables restantes; es decir, la adición de un nuevo artículo -que por sí solo representa un cambio en el nivel de estructura-, a su vez, influye en el nivel de entropía, debido a las palabras adicionadas en dicho artículo, y probablemente los nuevos elementos contengan referencias -ya sea internas o externas- que también modifican el nivel de interdependencia de la ley.

Reafirmando los postulados de Bourcier y Mazzega (2007: 3), el índice de Pearson demuestra que la correlación más estrecha entre variables es la existente entre el número de componentes (estructura) y la relación entre ellos (interdependencia), registrando un índice de correlación de 0.902 . 


\section{Conclusiones}

A través de la evaluación en el tiempo de la complejidad legal de la LIVA, mediante el análisis de su estructura, lenguaje e interdependencia se concluye lo siguiente:

El análisis de la estructura refleja que la LIVA de 2016, tanto en valores absolutos como logarítmicos, es más compleja que la versión original publicada en 1978. Esta complejidad se deriva de las adiciones y modificaciones realizadas al cuerpo de la ley desde su publicación y a lo largo de 37 años de vigencia de este ordenamiento legal. Esta afirmación se sustenta en que los tres indicadores analizados (estructura, entropía e interdependencia) muestran una tendencia generalizada al alza desde la entrada en vigor de la ley.

El análisis de entropía concluye que la LIVA en 2016 presentó una frecuencia relativa por cada palabra utilizada distinta a la que presentó la LIVA 1978, reflejada en la diferencia en extensión y la distribución de palabras. Esto se observa en el indicador de entropía obtenido para la LIVA de 2016 que fue de $8.9 \%$ en comparación con el 8.57\% registrado por la LIVA de 1978.

El análisis de interdependencia muestra que la LIVA 2016 contiene más de tres veces el número de referencias que la LIVA original de 1978, lo que significa que el proceso de lectura y entendimiento de la LIVA 2016 se hace más complejo, por el hecho de requerir la identificación y comprensión de más de tres veces el número de referencias publicadas en la LIVA original de 1978.

El análisis de complejidad legal conjunto con valores absolutos refleja que la LIVA en 2016 presenta una dificultad de comprensión y entendimiento de más de tres veces que lo registrado por la LIVA original de 1978, este incremento en el indicador de complejidad legal es resultado primordialmente de los cambios en la estructura y en el incremento de la interdependencia intra ley de la LIVA de 2016 en comparación con la ley original publicada en 1978.

Asimismo, el indicador conjunto de complejidad legal con valores logarítmicos mostró un valor de $6.10 \%$ en la LIVA DE 2016, 1.99\% mayor con respecto al nivel de complejidad de la ley publicada en 1978.

La innovación de esta entrega se encuentra en el estudio de la complejidad legal del universo de las versiones de la ley del IVA publicadas hasta ahora, caracterizando el comportamiento de esta variable a través del tiempo.

La construcción de este índice de complejidad legal, sin precedente en México, representa el primer esfuerzo por incursionar en este tema, sirviendo 
de base a futuras investigaciones que pretendan analizar la manera en que la complejidad de las leyes influye en los niveles de cumplimiento de las mismas.

A partir de lo anterior, en futuras investigaciones se deberá realizar la evaluación del impacto a través del tiempo de la complejidad legal en la recaudación del IVA en México, con el objeto de identificar alguna variable que permita superar el umbral hasta ahora registrado de la recaudación del IVA como porcentaje del PIB.

\section{Referencias}

Bourcier, D. y P. Mazzega (2007). “Toward measures of complexity in legal systems". Proceedings of the 11 th international conference on Artificial intelligence and law, 211-215. doi: 10.1145/1276318.1276359.

Epstein, R. (1997). "Simple rules for a complex world". Harvard University Press.

Kades, E. (1997). "The laws of complexity and the complexity of the laws: the implications of computational complexity theory of the law". William \& Mary Law School, 49, pp. 403-484.

Katz, D. (2010). Measuring the Complexity of the Law : The United States Code. Recuperado el 1 de mayo de 2016, de Computational Legal Studies: http://computationallegalstudies.com/2010/08/02/measuring-the-complexity-of-the-law-theunited-states-code/.

Katz, D. y M. Bommarito (2013). "Measuring the complexity of the law: the United States Code". Michigan State University.

Krever, R. y P. Mellor (2015). Where's the complexity in tax law? Recuperado el 1 de mayo de 2016, de University of Oxford: http://www.sbs.ox.ac.uk/sites/default/ files/Business_Taxation/Events/conferences/symposia/2015/krever-paper.pdf.

Long, S. y J. Swingen (1987). An Approach to the Measurement of Tax Law Complexity. Recuperado el 1 de mayo de 2016, de Journal of the American Taxation Association: http://connection.ebscohost.com/c/articles/6143625/approach-measurement-tax-law-complexity. 
McFedries, P. (2010). Excel 2010. (E. Tuya, ed. y D. Baeza, Trad.) Madrid, España: Grupo Anaya, pp. 88-130.

OCDE (2013). Revenue Statistics in Latin America. Organización para la Cooperación y Desarrollo Económicos, Economic Commission for Latin America and the Caribbean. OECD Publishing. Recuperado el 20 de julio de 2016, de http://www.oecd-ilibrary. org/taxation/revenue-statistics-in-latin-america-2014_9789264207943-en-fr.

Roberts, S.; W. Friedman, M. Ginsburg y M. Young (1972). “A report on complexity and the income tax". Tax Law Review, 3(27).

Ruhl, J. y D. Katz (2014). Measuring, monitoring and managing legal complexity. Recuperado el 1 de mayo de 2016, de Iowa Law Review: http://ilr.law.uiowa.edu/ files/ilr.law.uiowa.edu/files/ILR_101-1_Ruhl\%26Katz.pdf.

Schuck, P. (1992). "Legal complexity: some causes, consequences and cures". Duke Law Journal, 42(1), 1-52.

Waltl, B. y F. Matthes (2014). Towards measures of complexity: aplying structural and linguistic metrics to German laws. Recuperado el 2 de mayo de 2015, de Universidad de Munich: https://wwwmatthes.in.tum.de/file/1w4qwtvn2juy6/SebisPublic-Website/-/Towards-Measures-of-Complexity-Applying-Structural-andLinguistic-Metrics-to-German-Laws/Wa14c.pdf. 
Anexo 1: valores de estructura, entropía, interdependencia, complejidad y complejidad con logaritmos naturales de la ley del IVA

\begin{tabular}{|c|c|c|c|c|c|}
\hline Periodo & Estructura & Entropía & Interdep & Complejidad & \begin{tabular}{|c|} 
Complejidad \\
(Ln)
\end{tabular} \\
\hline 1T 1978 & 278 & 8.57 & 61 & 347.57 & 11.88 \\
\hline 2T 1978 & 278 & 8.57 & 61 & 347.57 & 11.88 \\
\hline 3T 1978 & 278 & 8.57 & 61 & 347.57 & 11.88 \\
\hline 4T 1978 & 278 & 8.57 & 61 & 347.57 & 11.88 \\
\hline 1T 1979 & 278 & 8.57 & 61 & 347.57 & 11.88 \\
\hline 2T 1979 & 278 & 8.57 & 61 & 347.57 & 11.88 \\
\hline 3T 1979 & 278 & 8.57 & 61 & 347.57 & 11.88 \\
\hline 4T 1979 & 278 & 8.57 & 61 & 347.57 & 11.88 \\
\hline 1T 1980 & 312 & 8.61 & 183 & 503.61 & 13.10 \\
\hline 2T 1980 & 312 & 8.61 & 183 & 503.61 & 13.10 \\
\hline 3T 1980 & 312 & 8.61 & 183 & 503.61 & 13.10 \\
\hline $4 \mathrm{~T} 1980$ & 312 & 8.61 & 183 & 503.61 & 13.10 \\
\hline 1T 1981 & 371 & 8.69 & 201 & 580.69 & 13.38 \\
\hline 2Т 1981 & 371 & 8.69 & 201 & 580.69 & 13.38 \\
\hline 3T 1981 & 371 & 8.69 & 201 & 580.69 & 13.38 \\
\hline 4T 1981 & 371 & 8.69 & 201 & 580.69 & 13.38 \\
\hline 1T 1982 & 359 & 8.64 & 193 & 560.64 & 13.30 \\
\hline 2Т 1982 & 359 & 8.64 & 193 & 560.64 & 13.30 \\
\hline 3T 1982 & 359 & 8.64 & 193 & 560.64 & 13.30 \\
\hline 4T 1982 & 359 & 8.64 & 193 & 560.64 & 13.30 \\
\hline 1T 1983 & 485 & 8.74 & 200 & 693.74 & 13.65 \\
\hline 2T 1983 & 485 & 8.74 & 200 & 693.74 & 13.65 \\
\hline 3T 1983 & 485 & 8.74 & 200 & 693.74 & 13.65 \\
\hline 4T 1983 & 485 & 8.74 & 200 & 693.74 & 13.65 \\
\hline 1T 1984 & 491 & 8.73 & 195 & 694.73 & 13.63 \\
\hline 2Т 1984 & 491 & 8.73 & 195 & 694.73 & 13.63 \\
\hline 3T 1984 & 491 & 8.73 & 195 & 694.73 & 13.63 \\
\hline 4T 1984 & 491 & 8.73 & 195 & 694.73 & 13.63 \\
\hline 1T 1985 & 491 & 8.73 & 195 & 694.73 & 13.63 \\
\hline 2Т 1985 & 491 & 8.73 & 195 & 694.73 & 13.63 \\
\hline 3T 1985 & 491 & 8.73 & 195 & 694.73 & 13.63 \\
\hline 4T 1985 & 491 & 8.73 & 195 & 694.73 & 13.63 \\
\hline 1T 1986 & 486 & 8.71 & 187 & 681.71 & 13.58 \\
\hline $2 \mathrm{~T} 1986$ & 486 & 8.71 & 187 & 681.71 & 13.58 \\
\hline 3T 1986 & 486 & 8.71 & 187 & 681.71 & 13.58 \\
\hline 4T 1986 & 486 & 8.71 & 187 & 681.71 & 13.58 \\
\hline 1T 1987 & 486 & 8.72 & 190 & 684.72 & 13.59 \\
\hline 2Т 1987 & 486 & 8.72 & 190 & 684.72 & 13.59 \\
\hline
\end{tabular}


Javier Moreno, Jaime H. Beltrán, Leovardo Mata

Anexo 1. Continuación.

\begin{tabular}{|c|c|c|c|c|c|}
\hline Periodo & Estructura & Entropía & Interdep & Complejidad & $\begin{array}{c}\text { Complejidad } \\
\text { (Ln) }\end{array}$ \\
\hline 3T 1987 & 486 & 8.72 & 190 & 684.72 & 13.59 \\
\hline 4T 1987 & 486 & 8.72 & 190 & 684.72 & 13.59 \\
\hline 1T 1988 & 496 & 8.75 & 201 & 705.75 & 13.67 \\
\hline 2Т 1988 & 496 & 8.75 & 201 & 705.75 & 13.67 \\
\hline 3T 1988 & 496 & 8.75 & 201 & 705.75 & 13.67 \\
\hline 4T 1988 & 496 & 8.75 & 201 & 705.75 & 13.67 \\
\hline 1T 1989 & 482 & 8.75 & 202 & 692.75 & 13.65 \\
\hline 2Т 1989 & 482 & 8.75 & 202 & 692.75 & 13.65 \\
\hline 3Т 1989 & 482 & 8.75 & 202 & 692.75 & 13.65 \\
\hline 4T 1989 & 482 & 8.75 & 202 & 692.75 & 13.65 \\
\hline $1 \mathrm{~T} 1990$ & 477 & 8.74 & 215 & 700.74 & 13.70 \\
\hline 2T 1990 & 477 & 8.74 & 215 & 700.74 & 13.70 \\
\hline 3T 1990 & 477 & 8.74 & 215 & 700.74 & 13.70 \\
\hline 4T 1990 & 477 & 8.74 & 215 & 700.74 & 13.70 \\
\hline 1T 1991 & 483 & 8.75 & 216 & 707.75 & 13.72 \\
\hline 2T 1991 & 483 & 8.75 & 216 & 707.75 & 13.72 \\
\hline 3Т 1991 & 483 & 8.75 & 216 & 707.75 & 13.72 \\
\hline 4T 1991 & 483 & 8.75 & 216 & 707.75 & 13.72 \\
\hline 1T 1992 & 417 & 8.70 & 212 & 637.70 & 13.55 \\
\hline 2Т 1992 & 417 & 8.70 & 212 & 637.70 & 13.55 \\
\hline 3Т 1992 & 420 & 8.70 & 213 & 641.70 & 13.56 \\
\hline $4 \mathrm{~T} 1992$ & 420 & 8.70 & 213 & 641.70 & 13.56 \\
\hline 1T 1993 & 420 & 8.70 & 213 & 641.70 & 13.56 \\
\hline 2T 1993 & 420 & 8.70 & 213 & 641.70 & 13.56 \\
\hline 3Т 1993 & 420 & 8.70 & 213 & 641.70 & 13.56 \\
\hline 4T 1993 & 420 & 8.70 & 213 & 641.70 & 13.56 \\
\hline 1T 1994 & 420 & 8.70 & 212 & 640.70 & 13.56 \\
\hline 2Т 1994 & 420 & 8.70 & 212 & 640.70 & 13.56 \\
\hline 3T 1994 & 420 & 8.70 & 212 & 640.70 & 13.56 \\
\hline 4T 1994 & 420 & 8.70 & 212 & 640.70 & 13.56 \\
\hline 1T 1995 & 443 & 8.75 & 220 & 671.75 & 13.65 \\
\hline 2T 1995 & 428 & 8.76 & 228 & 664.76 & 13.65 \\
\hline 3T 1995 & 428 & 8.76 & 228 & 664.76 & 13.65 \\
\hline $4 \mathrm{~T} 1995$ & 428 & 8.76 & 228 & 664.76 & 13.65 \\
\hline $1 \mathrm{~T} 1996$ & 439 & 8.84 & 233 & 680.84 & 13.71 \\
\hline 2Т 1996 & 439 & 8.84 & 233 & 680.84 & 13.71 \\
\hline 3Т 1996 & 439 & 8.84 & 233 & 680.84 & 13.71 \\
\hline $4 \mathrm{~T} 1996$ & 439 & 8.84 & 233 & 680.84 & 13.71 \\
\hline 1T 1997 & 448 & 8.86 & 231 & 687.86 & 13.72 \\
\hline 2Т 1997 & 448 & 8.86 & 231 & 687.86 & 13.72 \\
\hline 3Т 1997 & 450 & 8.86 & 239 & 697.86 & 13.76 \\
\hline 4T 1997 & 450 & 8.86 & 239 & 697.86 & 13.76 \\
\hline
\end{tabular}


Anexo 1. Continuación.

\begin{tabular}{|c|c|c|c|c|c|}
\hline Periodo & Estructura & Entropía & Interdep & Complejidad & $\begin{array}{c}\text { Complejidad } \\
\text { (Ln) }\end{array}$ \\
\hline $1 \mathrm{~T} 1998$ & 444 & 8.84 & 241 & 693.84 & 13.76 \\
\hline 2T 1998 & 444 & 8.84 & 241 & 693.84 & 13.76 \\
\hline 3T 1998 & 444 & 8.84 & 241 & 693.84 & 13.76 \\
\hline 4T 1998 & 444 & 8.84 & 241 & 693.84 & 13.76 \\
\hline $1 \mathrm{~T} 1999$ & 462 & 8.85 & 284 & 754.85 & 13.96 \\
\hline 2T 1999 & 462 & 8.85 & 284 & 754.85 & 13.96 \\
\hline 3T 1999 & 462 & 8.85 & 284 & 754.85 & 13.96 \\
\hline 4T 1999 & 462 & 8.85 & 284 & 754.85 & 13.96 \\
\hline $1 \mathrm{~T} 2000$ & 530 & 8.77 & 339 & 877.77 & 14.27 \\
\hline 2T 2000 & 530 & 8.77 & 339 & 877.77 & 14.27 \\
\hline 3T 2000 & 530 & 8.77 & 339 & 877.77 & 14.27 \\
\hline 4T 2000 & 530 & 8.77 & 339 & 877.77 & 14.27 \\
\hline $1 \mathrm{~T} 2001$ & 521 & 8.78 & 416 & 945.78 & 14.45 \\
\hline 2T 2001 & 521 & 8.78 & 416 & 945.78 & 14.45 \\
\hline 3T 2001 & 521 & 8.78 & 416 & 945.78 & 14.45 \\
\hline 4T 2001 & 521 & 8.78 & 416 & 945.78 & 14.45 \\
\hline 1 T 2002 & 521 & 8.78 & 416 & 945.78 & 14.45 \\
\hline 2Т 2002 & 521 & 8.78 & 416 & 945.78 & 14.45 \\
\hline 3 T 2002 & 521 & 8.78 & 416 & 945.78 & 14.45 \\
\hline $4 \mathrm{~T} 2002$ & 521 & 8.78 & 416 & 945.78 & 14.45 \\
\hline $1 \mathrm{~T} 2003$ & 530 & 8.89 & 423 & 961.89 & 14.50 \\
\hline 2Т 2003 & 530 & 8.89 & 423 & 961.89 & 14.50 \\
\hline $3 \mathrm{~T} 2003$ & 530 & 8.89 & 423 & 961.89 & 14.50 \\
\hline 4T 2003 & 530 & 8.89 & 423 & 961.89 & 14.50 \\
\hline $1 \mathrm{~T} 2004$ & 618 & 8.92 & 416 & 1042.92 & 14.64 \\
\hline $2 \mathrm{~T} 2004$ & 618 & 8.92 & 416 & 1042.92 & 14.64 \\
\hline 3Т 2004 & 618 & 8.92 & 416 & 1042.92 & 14.64 \\
\hline 4T 2004 & 618 & 8.92 & 416 & 1042.92 & 14.64 \\
\hline $1 \mathrm{~T} 2005$ & 679 & 8.90 & 492 & 1179.90 & 14.90 \\
\hline 2 T 2005 & 679 & 8.90 & 492 & 1179.90 & 14.90 \\
\hline 3Т 2005 & 681 & 8.90 & 477 & 1166.90 & 14.87 \\
\hline $4 \mathrm{~T} 2005$ & 681 & 8.90 & 477 & 1166.90 & 14.87 \\
\hline $1 \mathrm{~T} 2006$ & 623 & 8.86 & 527 & 1158.86 & 14.88 \\
\hline 2T 2006 & 623 & 8.86 & 527 & 1158.86 & 14.88 \\
\hline 3 T 2006 & 625 & 8.87 & 529 & 1162.87 & 14.89 \\
\hline 4T 2006 & 625 & 8.87 & 529 & 1162.87 & 14.89 \\
\hline $1 \mathrm{~T} 2007$ & 625 & 8.87 & 529 & 1162.87 & 14.89 \\
\hline 2Т 2007 & 625 & 8.87 & 529 & 1162.87 & 14.89 \\
\hline 3Т 2007 & 625 & 8.87 & 529 & 1162.87 & 14.89 \\
\hline 4T 2007 & 625 & 8.87 & 529 & 1162.87 & 14.89 \\
\hline $1 \mathrm{~T} 2008$ & 625 & 8.87 & 527 & 1160.87 & 14.88 \\
\hline 2T 2008 & 625 & 8.87 & 527 & 1160.87 & 14.88 \\
\hline
\end{tabular}




\section{Anexo 1. Conclusión.}

\begin{tabular}{|c|c|c|c|c|c|}
\hline Periodo & Estructura & Entropía & Interdep & Complejidad & $\begin{array}{c}\text { Complejidad } \\
\text { (Ln) }\end{array}$ \\
\hline 3Т 2008 & 625 & 8.87 & 527 & 1160.87 & 14.88 \\
\hline 4T 2008 & 625 & 8.87 & 527 & 1160.87 & 14.88 \\
\hline $1 \mathrm{~T} 2009$ & 625 & 8.87 & 527 & 1160.87 & 14.88 \\
\hline 2T 2009 & 625 & 8.87 & 527 & 1160.87 & 14.88 \\
\hline 3T 2009 & 625 & 8.87 & 527 & 1160.87 & 14.88 \\
\hline 4T 2009 & 625 & 8.87 & 527 & 1160.87 & 14.88 \\
\hline 1T 2010 & 625 & 8.87 & 529 & 1162.87 & 14.89 \\
\hline 2T 2010 & 625 & 8.87 & 529 & 1162.87 & 14.89 \\
\hline 3Т 2010 & 625 & 8.87 & 529 & 1162.87 & 14.89 \\
\hline 4T 2010 & 625 & 8.87 & 529 & 1162.87 & 14.89 \\
\hline $1 \mathrm{~T} 2011$ & 625 & 8.87 & 529 & 1162.87 & 14.89 \\
\hline 2T 2011 & 625 & 8.87 & 529 & 1162.87 & 14.89 \\
\hline 3T 2011 & 625 & 8.87 & 529 & 1162.87 & 14.89 \\
\hline 4 T 2011 & 625 & 8.87 & 529 & 1162.87 & 14.89 \\
\hline 1T 2012 & 625 & 8.87 & 529 & 1162.87 & 14.89 \\
\hline 2T 2012 & 625 & 8.87 & 529 & 1162.87 & 14.89 \\
\hline 3T 2012 & 625 & 8.87 & 529 & 1162.87 & 14.89 \\
\hline 4T 2012 & 625 & 8.87 & 529 & 1162.87 & 14.89 \\
\hline 1T 2013 & 625 & 8.87 & 529 & 1162.87 & 14.89 \\
\hline 2 T 2013 & 625 & 8.87 & 529 & 1162.87 & 14.89 \\
\hline $3 \mathrm{~T} 2013$ & 625 & 8.87 & 529 & 1162.87 & 14.89 \\
\hline $4 \mathrm{~T} 2013$ & 625 & 8.87 & 529 & 1162.87 & 14.89 \\
\hline 1T 2014 & 621 & 8.90 & 448 & 1077.90 & 14.72 \\
\hline 2T 2014 & 621 & 8.90 & 448 & 1077.90 & 14.72 \\
\hline 3T 2014 & 621 & 8.90 & 448 & 1077.90 & 14.72 \\
\hline 4T 2014 & 621 & 8.90 & 448 & 1077.90 & 14.72 \\
\hline 1T 2015 & 621 & 8.90 & 448 & 1077.90 & 14.72 \\
\hline 2T 2015 & 621 & 8.90 & 448 & 1077.90 & 14.72 \\
\hline 3Т 2015 & 621 & 8.90 & 448 & 1077.90 & 14.72 \\
\hline 4T 2015 & 621 & 8.90 & 448 & 1077.90 & 14.72 \\
\hline $1 \mathrm{~T} 2016$ & 621 & 8.90 & 448 & 1077.90 & 14.72 \\
\hline
\end{tabular}

Fuente: elaboración propia. 